\title{
ON A CONJECTURE IN THE THEORY OF PERMANENTS ${ }^{1}$
}

\author{
BY ALBERT NIJENHUIS AND HERBERT S. WILF
}

Communicated by Gian-Carlo Rota, March 13, 1970

Let $A$ be an $n \times n$ matrix of zeros and ones, with $r_{i}$ ones in the $i$ th row $(i=1, \cdots, n)$. It has been conjectured $[1]$ that

$$
\text { Per } A \leqq \prod_{i=1}^{n} r_{i} !^{1 / r_{i}}
$$

which, if true, would be best possible. We sketch here a proof of the fact that

$$
\text { Per } A \leqq \prod_{i=1}^{n}\left\{\boldsymbol{r}_{i} !^{1 / r_{i}}+\tau\right\}
$$

where $\tau=.1367 \ldots$ is a universal constant. Details of the proof will appear elsewhere [2].

Suppose $\phi$ is a function of the positive integers for which $\phi(1)=1$ and

$$
\text { Per } A \leqq \prod_{i=1}^{n-1} \phi\left(r_{i}\right)
$$

is true for all $(n-1) \times(n-1)$ matrices $A$. If now $A$ is $n \times n$, expanding by minors down some column, one finds that (3) holds with $n$ replacing $n-1$ provided that

$$
\sum_{i=1}^{c} \frac{1}{\phi\left(r_{i}-1\right)} \prod_{k=1}^{c} \frac{\phi\left(r_{k}-1\right)}{\phi\left(r_{k}\right)} \leqq 1
$$

for all positive integers $c$ and $r_{1}, \cdots, r_{c} \geqq 2$. Consider the function $\phi$ defined recursively by

$$
\begin{aligned}
& \text { (a) } \phi(1)=1 \\
& \text { (b) } \phi(n+1)=\phi(n) \exp [1 / e \phi(n)] \quad(n \geqq 1) .
\end{aligned}
$$

Substituting (5) into (4) one finds easily that (4) holds.

By rather lengthy arguments we prove that for the $\phi$ of (5) we have

$$
\phi(n)=\frac{n}{e}+\frac{\log n}{2 e}+\frac{A}{e}+o(1) \quad(n \rightarrow \infty)
$$

AMS Subject Classifications. Primary 0525, 0515; Secondary 4150.

Key Words and Phrases. Permanent, matrix of zeros and ones, asymptotic expansion.

Research supported in part by the National Science Foundation. 
and

(7)

$$
\phi(n) \leqq n !^{1 / n}+\frac{A-\log \sqrt{ }(2 \pi)}{e} \quad(n=1,2,3, \cdots)
$$

which together prove (2) with $\tau=(A-\log \sqrt{ }(2 \pi)) / e$.

\section{REFERENCES}

1. H. Minc, Upper bounds for permanents of $(0,1)$-matrices, Bull. Amer. Math. Soc. 69 (1963), 789-791. MR $27 \# 5777$.

2. A. Nijenhius and H.S. Wilf, On a conjecture of Ryser and Minc, Nederl. Akad. Wetensch. Proc. Ser. A 73 (1970), 151-157.

University of Pennsylvania, Philadelphia, Pennsylvania 19104 